\title{
AN ANALYSIS ON GAME THEORETIC NEGOTIATION DYNAMISM BASED ON MULTI-AGENT PARADIGM IN VIRTUAL ENTERPRISE
}

\author{
Toshiya Kaihara ${ }^{1}$ and Susumu Fujii ${ }^{2}$ \\ ${ }^{1}$ Kobe University, Graduate School of Science and Technology \\ ${ }^{2}$ Kobe University, Department of Computer and Systems Engineering \\ \{kaihara,fujii\}@ms.cs.kobe-u.ac.jp
}

\begin{abstract}
In this paper, we focus on negotiation process in VE formulation as a basic research to clarify its effective management. Each enterprise in $V E$ is defined as agent in multi-utilities and a framework of multi-agent programming with game theoretic approach is newly proposed as negotiation algorithm amongst the agents. Each unit is defined as agent in our VE model, and their decision makings are formulated as a game theoretic methodology. We adopt CNP (Contract Net Protocol) as the coordination and negotiation mechanism amongst the units. CNP models transfer of control in a distributed system with the metaphor of negotiation among autonomous intelligent beings. CNP consists of a set of nodes that negotiate with one another through a set of message. Nodes generally represent the distributed computing resources to be managed, correspond to "enterprises" in this paper. We develop a computer simulation model to form VE through multiple negotiations anongst several potential members in the negotiation domain, and finally clarify the formulation dynamism with the negotiation process.
\end{abstract}

\section{INTRODUCTION}

Nowadays, Virtual Enterprise (VE) is a crucial paradigm of business management in agile environment. VE exists in both service and manufacturing organizations, although the complexity of the each enterprise in VE may vary greatly from industry to industry. Realistic VE handles multiple end products with shared components, facilities and capacities (Camarinha-Matos, 1999). Since the flow of materials in VE is not always along an arborescent network, various modes of transportation may be considered, and the bill of materials for the end items may be both deep and large.

Traditionally, marketing, distribution, planning, manufacturing, and the purchasing organizations operated independently. These organizations have their own objectives and these are often conflicting. Marketing's objective of high customer service and maximum sales conflict with manufacturing and distribution goals. Many manufacturing operations are designed to maximize throughput and lower costs with little consideration for the impact on inventory levels and 
distribution capabilities. Purchasing contracts are often negotiated with very little information beyond historical buying patterns. The result of these factors is that there is not a single, integrated plan for the organization - there were as many plans as businesses. Clearly, there is a need for a mechanism through which these different functions can be integrated together. Although cooperation is the fundamental characteristic of VE concept, due to its distributed environment and the autonomous and heterogeneous nature of the VE members, cooperation can only be succeed if a proper management of dependencies between activities is in place just like Supply Chain Management (Fisher, 1994) (Goldratt, 1983).

In this paper, we focus on negotiation process in VE formulation as a basic research to clarify its effective management. Each enterprise in VE is defined as agent with multi-utilities and a framework of multi-agent programming with game theoretic approach (Von Neumann, 1947) is newly proposed as negotiation algorithm amongst the agents. Each unit is defined as agent in our VE model, and their decision makings are formulated as a game theoretic methodology. We adopt CNP (Contract Net Protocol) (Smith, 1980) (Durfee, 1987) as the coordination and negotiation mechanism amongst the units. CNP models transfer of control in a distributed system with the metaphor of negotiation among autonomous intelligent beings. CNP consists of a set of nodes that negotiate with one another through a set of message (Kaihara, 2002a, 2002b). Nodes generally represent the distributed computing resources to be managed, correspond to "enterprises" in this paper. We develop a computer simulation model to form VE through multiple negotiations amongst several potential members in the negotiation domain, and finally clarify the formulation dynamism with the negotiation process.

\section{ENTERPRISE AGENT}

\subsection{Virtual Enterprise Model}

A large number of diversified networked organisations of enterprises fall under the general definition of VE. We assumed our VE model in the possible simplest definition as a basic research, as follows:

i) Duration: Single business

An alliance of the enterprises is established towards a single business opportunity, and is dissolved at the end of such process.

ii) Topology: Fixed structure

There exist established supply chains with an almost fixed structure.

iii) Participation: Single alliance

All the enterprises are participating into only a single alliance at the same time.

iv) Coordination: Democratic alliance

A different organisation can be found in some supply chains without a dominant company. All the enterprises cooperate on an equal basis, preserving their autonomy.

v) Visibility scope: Single level

All the enterprises in VE communicate only to its direct neighbours in its architecture (figure 1). That is the case observed in most supply chains. 


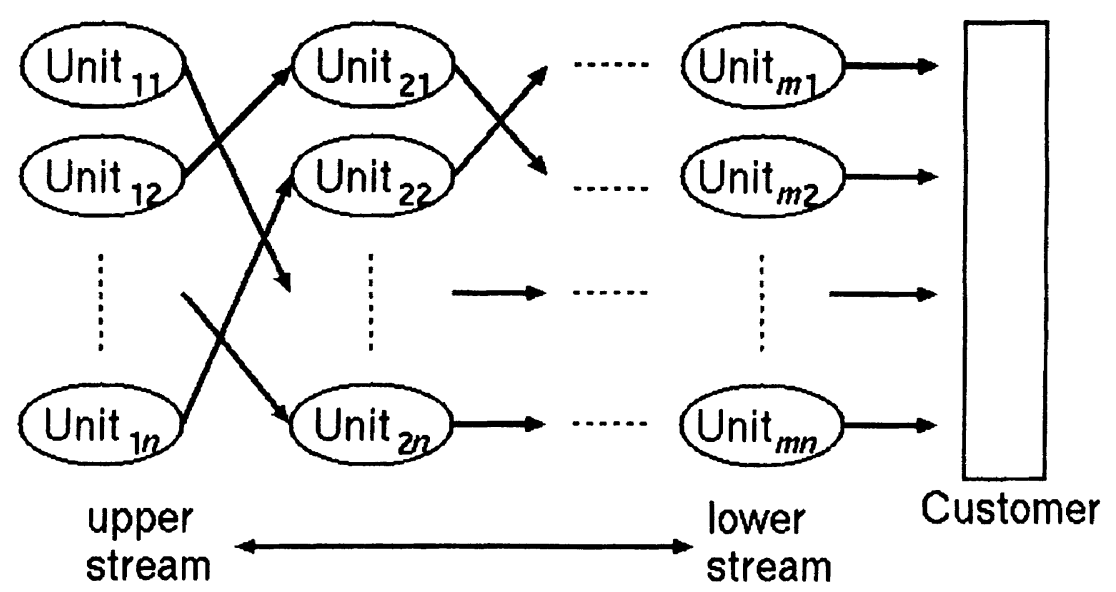

Figure 1 VE model

Figure 1 shows the assumed VE model in this paper. We call an enterprise as unit, and there exist $m$ layers, which have $m_{n}$ units in the VE model. The lowest level corresponds to consumers who can create original task requests to the VE. As the layer number, $m$, increases, we describe it 'lower' based on the product flow order in this paper.

At first, the customer dispatches new order to all the units in layer $m$, and then several units, which are satisfied with the order, responds and circulates the order toward upper units in the VE model. Finally a VE with single supply chain will be established for the order as a consequence of their negotiations through all the layers.

\subsection{Unit Structure}

Each unit is defined as agent in our VE model, and its structure is described in figure 2. We adopt CNP as the coordination and negotiation mechanism amongst the units. CNP models transfer of control in a distributed system with the metaphor of negotiation among autonomous intelligent beings. CNP consists of a set of nodes that negotiate with one another through a set of message. Nodes generally represent the distributed computing resources to be managed, correspond to "units" in this paper.

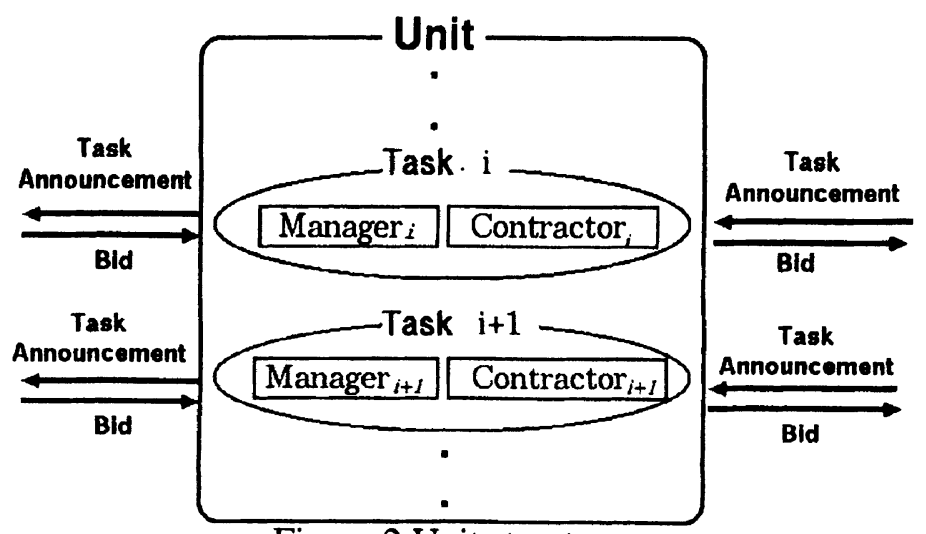

Figure 2 Unit structure 
An agent (=unit) can act both as a manager and a contractor of a delivery sets. When a unit receives new order ( $=$ task announcement) $i$, it creates a contractor / manager set (Manager $i$ / Contractor $i$ ) for the task inside. Manager $i$ creates a new order towards the lower units to secure the contract with the upper layer.

\subsection{Basic Assumptions}

There exist several situations in partnering amongst enterprise agents. In this paper it is assumed that the product demand is predictable in the negotiation under multipurpose criterion. That means order patterns are previously given and the negotiations start after the order reached to each enterprise agent. They should prepare robust solutions with maximum utilities against the order. We propose agent behaviours based on game theoretic approach according to this assumption.

\subsection{Negotiation Algorithm}

Negotiation steps according to agent roles are described as follows:

\section{Manager}

Step M1: Create a new task based on the received bid information.

Step M2: Task announcement (TA) to the lower units.

Step M3: After the bidding period expired, check all the acquired bids according to its standard. If there exists no bid to select, go to M4. Otherwise go to M5.

Step M4: Modify the task and go to M2.

Step M5: Select the task and send reward (Reward) to the corresponding unit.

\section{Contractor}

Step C1: Create an estimated bid.

Step C2: Send the bid.

Step C3: Request task announcement to the manager.

\subsection{Agent Behaviour}

In this model, all the orders are clearly given before the negotiations. Agent behaviours are described in each negotiation step.

\section{- Bidding (Step C2)}

Each contractor $\left(U_{i j}\right)$ has three attributes, such as cost, lead time and quality, in their bid for order $k$ defined as follows:

$$
\begin{aligned}
& \text { Cost }_{i j}^{k}=E_{i j}^{k}+D_{i j}^{k}+P_{i j}^{k} \\
& \text { Leadtime }{ }_{i j}^{k}=\lambda_{i j}^{k} / E_{i j}^{k} \\
& \text { Quality }_{i j}^{k}=\mu_{i j}^{k} D_{i j}^{k}\left(1-\exp ^{-v_{i j}^{k} P_{i j}^{k}}\right)
\end{aligned}
$$


where

Cost ${ }_{i j}^{k}$ : total cost for $U_{i j}$ to process order $k$

Leadtime ${ }_{i j}^{k}$ : lead time for $U_{i j}$ to process order $k$

Quality ${ }_{i j}^{k}$ : product quality for $U_{i j}$ to process order $k$

$E_{i j}^{k}, D_{i j}^{k}, P_{i j}^{k}$ : equipment / development / personnel cost

$\lambda_{i j}^{k}:$ coefficient of leadtime

$\mu_{i j}^{k}, v_{i j}^{k}:$ coefficients of quality

Cost vs. lead time, and cost vs. quality, are in trade off relationship in those equations with reality.

\section{- Reward (Step M5)}

After the bidding by contractors, managers compute following pay-off matrix according to their utilities against all the bids.

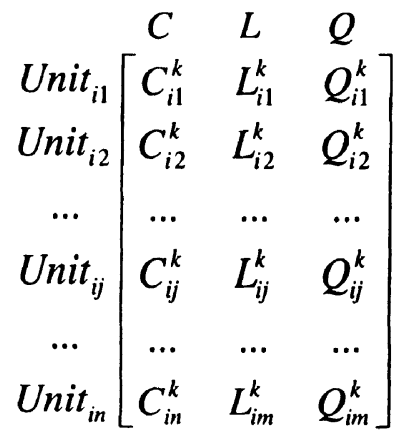

where

$$
\begin{aligned}
& C_{i j}^{k}=\left(\overline{c_{i}^{k}}-\text { Cost }_{i j}^{k}\right) / s_{c_{i}^{k}} \\
& L_{i j}^{k}=\left(\overline{l_{i}^{k}}-\text { Leadtime }_{i j}^{k}\right) / s_{l_{i}^{k}} \\
& Q_{i j}^{k}=\left(\text { Quality }_{i j}^{k}-\overline{q_{i}^{k}}\right) / s_{q_{i}^{k}} \\
& C_{i j}^{k}: \text { utility on cost for } U_{i j} \text { to process order } k \\
& L_{i j}^{k}: \text { utility on lead time for } U_{i j} \text { to process order } k \\
& \frac{Q_{i j}^{k}}{c_{i}^{k}}, \overline{l_{i}^{k}}, \overline{q_{i}^{k}}: \text { average utility on quality for } U_{i j} \text { to process order } k \\
& s_{c_{i}^{k}}, s_{l_{i}^{k}}, s_{q_{i}^{k}}: \text { standard deviation of all the bid on cost, lead time, quality }
\end{aligned}
$$

Five strategies are defined as selection mechanism using the payoff matrix in (4). - method 1: cost minimisation

$$
\max _{j=1,2, \ldots, n}=C_{i j}^{k}
$$


- method 2: lead time minimisation

$$
\max _{j=1,2, \ldots, n}=L_{i j}^{k}
$$

- method 3: quality maximisation

$$
\max _{j=1,2, \ldots, n}=Q_{i j}{ }^{k}
$$

- method 4: total utility maximisation

$$
\max _{j=1,2, \ldots, n}=C_{i j}^{k}+L_{i j}^{k}+Q_{i j}^{k}
$$

- method 5: max-min strategy

$$
\max _{j=1,2, \ldots, n} \min \left\{C_{i j}^{k}, L_{i j}^{k}, Q_{i j}^{k}\right\}
$$

Method 5 is so called " max-min strategy" in game theory, which has been proved to conduct Nash equilibrium solution by min-max theorem, if the game is in zero-sum situation like our formulation shown in (4).

\section{EXPERIMENTAL RESULTS}

\subsection{Simulation Model}

A 3-layered VE model for computer simulation was developed to clarify VE formulation dynamism with the proposed negotiation mechanism. Each layer consists of 5 enterprises in this simulation model described in Figure 1.

Simulation parameters are shown in Table 1 . All the results are the average of 500 trials in each simulation scenario.

Table 1 Simulation parameters

\begin{tabular}{|c|c|c|c|c|c|c|c|c|}
\hline$m$ & $n$ & $N$ & $E$ & $D$ & $P$ & $\lambda$ & $\mu$ & $v$ \\
\hline 3 & 5 & 1 & $5-15^{*}$ & $5-15^{*}$ & $5-15^{*}$ & 300 & 5 & 0.1 \\
\hline
\end{tabular}

* followed by uniformed random distribution

Table 2 Simulation results (Negotiation attributes)

\begin{tabular}{|c|c|c|c|c|c|c|c|c|c|c|}
\hline & \multicolumn{2}{|c|}{ Method 1 } & \multicolumn{2}{c|}{ Method 2 } & \multicolumn{2}{c|}{ Method 3 } & \multicolumn{2}{c|}{ Method 4 } & Method 5 \\
\hline & Ave. & $\begin{array}{c}\text { St. } \\
\text { Dev. }\end{array}$ & Ave. & $\begin{array}{c}\text { St. } \\
\text { Dev. }\end{array}$ & Ave. & $\begin{array}{c}\text { St. } \\
\text { Dev. }\end{array}$ & Ave. & $\begin{array}{c}\text { St. } \\
\text { Dev. }\end{array}$ & Ave. & $\begin{array}{c}\text { St. } \\
\text { Dev. }\end{array}$ \\
\hline Cost & 72.87 & 5.08 & 99.89 & 7.47 & 104.17 & 6.61 & 98.96 & 8.79 & 91.19 & 5.54 \\
\hline LTime & 119.53 & 18.47 & 68.32 & 4.84 & 90.21 & 18.06 & 80.72 & 11.04 & 91.04 & 12.24 \\
\hline Quality & 65.10 & 10.58 & 92.59 & 18.05 & 132.34 & 12.44 & 106.84 & 20.22 & 93.23 & 12.97 \\
\hline
\end{tabular}


Table 3 Simulation results (Utilities)

\begin{tabular}{|c|c|c|c|c|c|}
\hline & Method 1 & Method 2 & Method 3 & Method 4 & Method 5 \\
\hline$E_{i j}{ }^{k}$ & 8.13 & 13.35 & 9.96 & 11.68 & 10.38 \\
\hline$D_{i j}{ }^{k}$ & 8.13 & 10.11 & 12.84 & 11.47 & 10.61 \\
\hline$P_{i j}{ }^{k}$ & 8.03 & 9.84 & 11.92 & 9.83 & 9.41 \\
\hline
\end{tabular}

Simulation results in terms of negotiation attributes and utilities are shown in Table 2, 3, respectively. All the results are shown in the average (AVE.) and the standard distribution (St. Dev.) in Table 2. Figure 3 also illustrates the average of each negotiation attribute to compare the proposed methods.

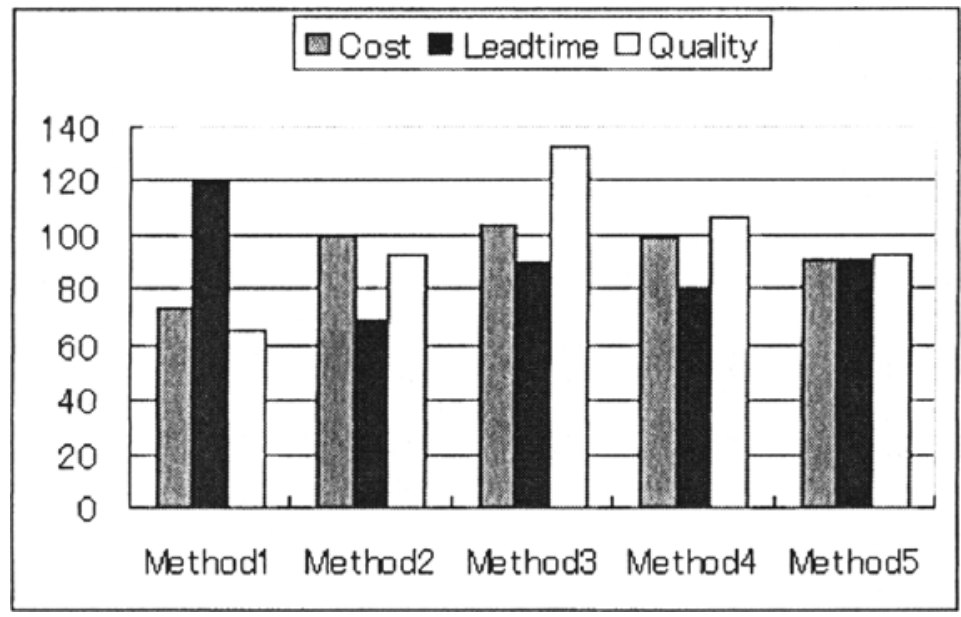

Figure 3 Unit structure

We summarise the characteristics of each method as follows:

Method 1: Cost minimisation

Since the negotiation amongst enterprises is cost-oriented in this method, cost parameter is the best of all the methods in Ave. Additionally Cost and Quality are better in St. Dev., because they are correlated to $E_{i j}^{k}, D_{i j}^{k} ; P_{i j}^{k}$ shown in (1) and (3). It has been observed that all the utilities are small to minimise total cost in this method at Table 3.

Method 2: Lead time minimisation

Lead time-oriented method naturally conducts the minimal LeadTime in Ave. and St. Dev. Cost parameter is not good, because Leadtime and $E_{i j}^{k}$ is in tradeoff relation in (2), and that lead to higher cost.

Method 3: Quality maximisation

It is obvious that quality maximisation strategy caused the worst in Cost, and this result fits well to our general sense. In this method enterprises don't pay any attention to LeadTime shown in (2).

Method 4: Total utility maximisation Generally the result is moderate in the balance amongst 3 parameters by trying to maximise total utility. In Figure 3 it has been observed that they are relatively 
better in its LeadTime and Quality, but worse in its Cost. That is because the general relationship amongst $E_{i j}^{k}, D_{i j}^{k}, P_{i j}^{k}$, in (1), (2) and (3), that means enterprise agents sacrifice LeadTime to increase Cost and Quality.

Method 5: Max-min strategy

Acquired result is completely well-balanced in Ave. It has also been confirmed that this strategy conducts minimal in St. Dev., and their negotiation is stable and robust enough to deal with agile trading situations. That is because each agent tried to minimise the risk based on the min-max theory.

Although our investigations have clearly not been exhaustive, it is already apparent that the agent behaviours have a great influence on autonomously formulated VE structure as a basic study. Proposed game theoretic formulation on agent decision mechanism with multi-agent paradigm is quite reasonable to realise negotiation process amongst enterprises.

\section{CONCLUSIONS}

In this paper, we focused on negotiation process in VE formulation as a basic research. Each enterprise in VE was defined as agent with multi-utilities and a framework of multi-agent programming with game theoretic is newly proposed as negotiation algorithm amongst the agents. Each unit is defined as agent in our VE model, and their decision-makings are formulated as a game theoretic methodology. Simulation results have proved that the proposed game theoretic formulation on agent decision mechanism with multi-agent paradigm is quite reasonable to analyse negotiation process amongst enterprises.

\section{ACKONWLEDGEMENTS}

This research was supported by International Research program IMS (Intelligent Manufacturing System) of MITI Japan, under contract No.0119 (HUTOP project).

\section{REFERENCES}

1. Camarinha-Matos, L. M. et al., The virtual enterprise concept, Infrastructures for virtual enterprises, Kluwer academic publishers, Boston, pp.3-14, 1999.

2. Durfee, E. et al., Coherent cooperation among communication problem solvers, IEEE Transaction on Computers, N 36, pp.1275-1291, 1987.

3. Fisher M. L., Making supply meet demand in uncertain world, Harvard Business Review, May/Jun, 1994.

4. Goldratt E. M., The GOAL, North River Press, 1983.

5. Kaihara, T. and S. Fujii, A proposal on negotiation methodology in VE, Collaborative Business Ecosystems and Virtual Enterprises, Kluwer Academic Publishers, Boston, pp125-132, 2002a.

6. Kaihara, T. and S. Fujii, IT based Virtual Enterprise Coalition Strategy for Agile Manufacturing Environment, Proc. of the 35th CIRP Int. Seminar on Manufacturing Systems, pp32-37, 2002b.

7. Von Neumann, J. et al., Theory of Games and Economic Behavior, Princeton University Press, 1947.

8. Smith, R., The contract net protocol, IEEE Transaction on Computers, C-29, pp.1104-1113, 1980. 